\title{
Palliative hysterectomy for vaginal bleeding from breast cancer metastatic to the uterus
}

\author{
Amnon A Berger ${ }^{1}$, Cathleen E Matrai ${ }^{2}$, Tessa Cigler ${ }^{2}$ and Melissa K Frey²

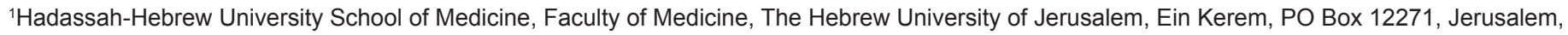 \\ 9112102, Israel \\ ${ }^{2}$ Weill Cornell Medicine, New York, NY 10065, USA
}

Correspondence to: Amnon A Berger. Email: amnon.berger@mail.huji.ac.il

\begin{abstract}
Breast cancer is the most prevalent cancer in the United States. With an increasing rate of survivorship and extended life span for patients with metastatic disease, the demand for palliative care is increasing. Although uncommon, metastases to gynaecologic organs have been reported and are often present with post-menopausal bleeding. Post-menopausal bleeding can become clinically significant and have a detrimental effect on quality of life. We report the case of a 70-year-old woman with symptomatic vaginal bleeding caused by breast cancer metastatic to her uterus, cervix, fallopian tubes and ovaries. She was successfully treated with minimally invasive hysterectomy, resolving her vaginal bleeding and anemia and allowing her to resume chemotherapy.
\end{abstract}

Keywords: breast neoplasms, endometrium, uterine bleeding, hysterectomy, anaemia, palliative care

ecancer 2018, 12:811 https://doi.org/10.3332/ecancer.2018.811

Copyright: $\odot$ the authors; licensee ecancermedicalscience. This is an Open Access article distributed under the terms of the Creative Commons Attribution License (http://creativecommons.org/licenses/by/3.0), which permits unrestricted use, distribution, and reproduction in any medium, provided the original work is properly cited. 


\section{Introduction and discussion}

Breast cancer is the most prevalent cancer in the USA with an estimated 252,710 new diagnoses in 2017 and 40,610 recorded deaths. Six per cent of patients have distant metastases at the time of diagnosis [1]. Breast cancer survivorship has been steadily increasing over the past several decades, owing to both earlier diagnoses and multiple treatment options, making breast cancer survival rates higher than most other cancer types [2]. The 5-year, 10-year and 15-year relative survival rates for breast cancer are $89 \%$, $83 \%$ and $79 \%$, respectively, leading to an estimated prevalence of more than 3.5 million women in USA with a history of breast cancer [3]. This improving survivorship results in an increased number of women living with metastatic breast cancer, with approximately 154,794 such women currently alive in the USA [4]. The increasing number of women living with metastatic disease requires continued attention and innovation in palliative care.

The most common sites of breast cancer metastases are the bone, lung, pleura, soft tissue and liver [5]. Metastases to the genital tract are rare; cancer that invades the uterus usually does so by direct extension and distant metastases are seldom found. However, when metastases to the uterus from remote origins are discovered, breast cancer is the most commonly discovered cancer (46.5\% of cases) [6]. Although lobular invasive carcinoma is more likely to present with distant metastases to the female genital tract, invasive ductal carcinoma has been reported [7]. Breast cancer cells have been discovered in the myometrium and endometrium [6] as well as in endometrial polyps [8] and, rarely, even inside the leiomyosarcoma of the uterus [9, 10]. Although uncommon, metastases to the uterine corpus are an established pathological phenomenon. Uterine metastases are most likely to involve the myometrium and remain asymptomatic for a relatively long duration; however, when the endometrium is involved, vaginal bleeding can occur [11, 12, 13].

We report the case of a 70-year-old gravida seven para five female, with a history of metastatic lobular breast cancer, positive for estrogen receptor $(E R)$ and progesterone receptor $(P R)$ staining, and negative for human epidermal growth factor receptor 2 staining. Her breast cancer was initially diagnosed 5 years prior, and at the time of diagnosis involved the axilla and bones.

The patient received multiple treatments, including Letrozole, Zoledronic Acid, Exemestane, Fulvestrant and Vinorelbine. Approximately 5 years after her cancer diagnosis, while being treated with Capecitabine, she developed vaginal bleeding. She underwent a pelvic exam, which was notable for a small mobile uterus, without active vaginal bleeding or adnexal masses. PET CT scan demonstrated progression of the disease in her bone (axial and proximal appendicular skeleton) and mediastinal lymph nodes and moderate fluorodeoxyglucose (FDG) uptake of the uterus centrally that was not present on imaging performed ten months prior (Figure 1). The patient underwent uterine dilatation and curettage and hysteroscopy. The intra-operative findings were notable for a large friable polypoid mass filling the endometrial cavity and pathologic examination of the endometrial mass revealed metastatic breast cancer (Figures 2 and 3 ).

Following the procedure, the patient's vaginal bleeding resolved, and she was started on Navelbine. As the patient's bleeding had resolved and she required systemic chemotherapy, no further gynaecologic procedure was planned. However, 3 months later, she presented to the emergency room with heavy vaginal bleeding and symptomatic anemia (hemoglobin $6.7 \mathrm{mg} / \mathrm{dl}$ and hematocrit $21.7 \mathrm{~g} / \mathrm{dl}$ ). She received a transfusion of packed red blood cells, but continued to experience vaginal bleeding. After a thorough review of her clinical status, prognosis, and the management options, she elected to proceed with a palliative hysterectomy. The patient underwent robotic-assisted total laparoscopic hysterectomy and bilateral salpingo-oophorectomy. The patient tolerated the procedure well with minimal blood loss and was discharged home on the same day. The final pathologic examination demonstrated metastatic breast cancer present in the bilateral ovaries, fallopian tubes, uterus (endometrium, myometrium and serosa) and cervix. The patient recovered well from surgery and continued chemotherapy. Her hemoglobin and hematocrit at her post-operative visit were $9.2 \mathrm{mg} / \mathrm{dl}$ and $27.8 \mathrm{~g} / \mathrm{dl}$, respectively. At 8 months following the surgery, the patient had experienced no further episodes of vaginal bleeding or anemia. 


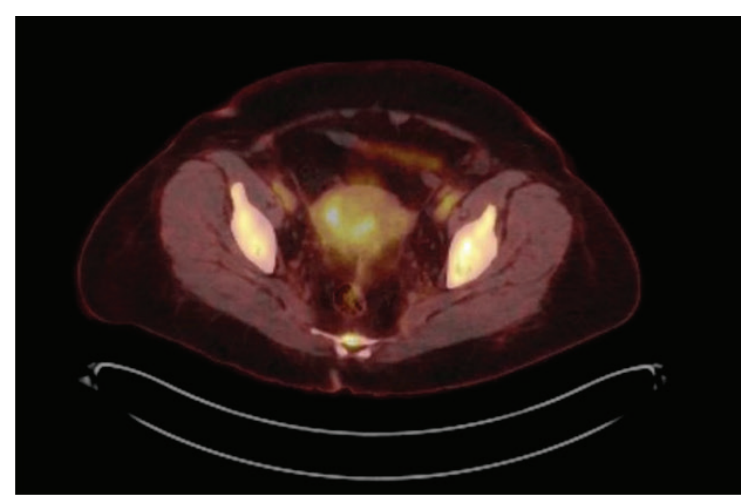

Figure 1. Increased FDG uptake on PET CT including uptake in uterus. PET CT scan. 1. Interval increase in FDG uptake of multiple hypermetabolic osseous metastases throughout the axial and proximal appendicular skeleton. 2. Mildly increased FDG uptake of mildly hypermetabolic mediastinal lymph nodes. 3. Moderate FDG uptake of the uterus centrally that was not present on imaging performed ten months prior.

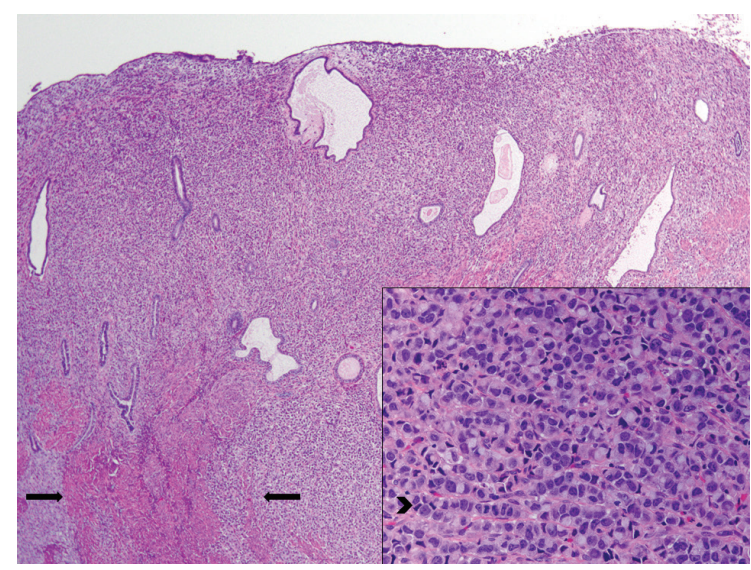

Figure 2. Breast metastasis in uterus corpus, endometrium and myometrium. Low-power image of uterus. There are sheets of neoplastic cells replacing the endometrial stroma and intersecting between muscle fibres of the myometrium (arrow). The tumour extended through the entire thickness of the myometrium to the uterine serosa. Cervical and adnexal involvement was also present. On high power (inset), the cells display areas of a classic invasive lobular carcinoma growth pattern, with single-filing of cells readily observed (arrowhead). 


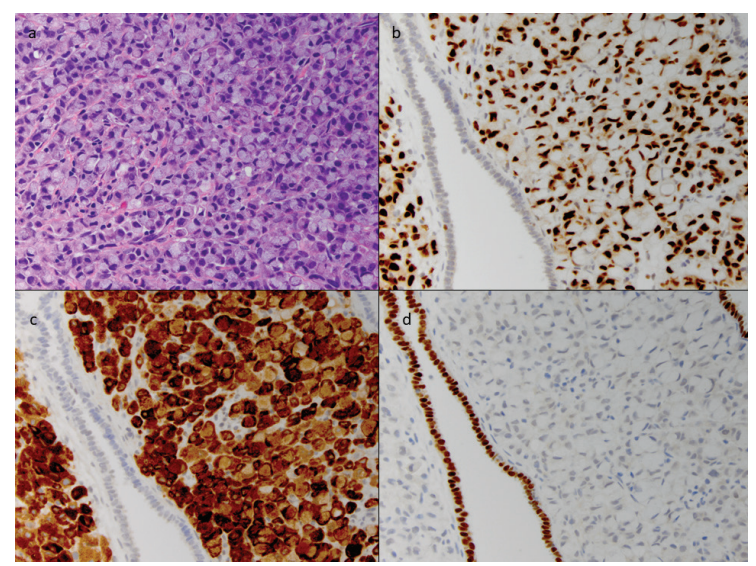

Figure 3. Cellular classification of tumour with immunohistochemistry. A high-power image of the tumour cells (a) shows abundant intracellular mucin displacing the nucleus to the periphery of the cell, imparting a 'signet ring' appearance. This feature was also noted in the prior axillary specimen. The tumour cells are strongly positive for GATA3 (b) and mammoglobin (c), two markers of breast origin, while the adjacent endometrium is negative. The cells do not stain for Pax-8 (d), a marker of Mullerian origin, while the intervening endometrium serves as a strong positive control. Additionally, approximately $1-10 \%$ of the tumour cells showed weak ER staining and less than $1 \%$ were positive for PR (not shown).

\section{Conclusions}

With the increasing prevalence of breast cancer, improvements in detection and the expanding spectrum of therapeutic options, a greater need has arisen for palliative care. Here, we present a case of rare metastases from breast cancer to the uterine corpus and endometrium that resulted in heavy vaginal bleeding and symptomatic anemia, and was successfully treated with minimally invasive hysterectomy. With the decreasing risk and morbidity of surgery owing to advancing surgical techniques, surgical palliation becomes an increasingly appealing option for some patients. We show that hysterectomy can provide long-term palliation for vaginal bleeding due to metastatic disease and should be considered for good surgical candidates when appropriate.

\section{References}

1. Howlader N, Noone AM, and Krapcho M, et al (eds) (2017) SEER Cancer Statistics Review, 1975-2014, National Cancer Institute [https://seer.cancer.gov/csr/1975 2014/] Date accessed: 01/07/17

2. Giordano SH, Buzdar AU, and Smith TL, et al (2004) Is breast cancer survival improving? Cancer 100(1) 44-52 https://doi. org/10.1002/cncr.11859

3. Miller KD, Siegel RL, and Lin CC, et al (2016) Cancer treatment and survivorship statistics CA Cancer J Clin 66(4) 271-289 https:// doi.org/10.3322/caac.21349 PMID: 27253694

4. Mariotto $A B$, Etzioni $R$, and Hurlbert $M$, et al (2017) Estimation of the number of women living with metastatic breast cancer in the United States Cancer Epidemiol Biomarkers Prev 26(6) 809-815 https://doi.org/10.1158/1055-9965.EPI-16-0889 PMID: 28522448

5. Lee YT (1983) Breast carcinoma: pattern of metastasis at autopsy J Surg Oncol 23(3) 175-180 https://doi.org/10.1002/ jso.2930230311 PMID: $\underline{6345937}$

6. Kumar NB and Hart WR (1982) Metastases to the uterine corpus from extragenital cancers. a clinicopathologic study of 63 cases Cancer 50(10) 2163-2169 PMID: 7127256 
7. Arslan D, Tural D, and Tatlı AM, et al (2013) Isolated uterine metastasis of invasive ductal carcinoma Case Rep Oncol Med 2013 793418 PMID: 23573438 PMCID: 3612463

8. Martinez MR, Marazuela MA, and Vallejo MR, et al (2016) Metastasis of lobular breast cancer to endometrial polyps with and without the presence of vaginal bleeding Int J Gynaecol Obstet 134(1) 101-102 https://doi.org/10.1016/j.ijgo.2016.01.005 PMID: $\underline{27045079}$

9. Yasuhara Y, Mikami Y, and Ishiguro S (2008) Metastatic breast carcinoma identified in a uterine leiomyosarcoma Pathol Int 58(5) 317-321 https://doi.org/10.1111/j.1440-1827.2008.02230.x PMID: 18429832

10. van Meurs HS, Dieles JJ, and Stel HV (2012) A uterine leiomyoma in which a leiomyosarcoma with osteoclast-like giant cells and a metastasis of a ductal breast carcinoma are present Ann Diagn Pathol 16(1) 67-70 https://doi.org/10.1016/j.anndiagpath.2010.11.010

11. Bezpalko K, Mohamed MA, and Mercer L, et al (2015) Concomitant endometrial and gall bladder metastasis in advanced multiple metastatic invasive lobular carcinoma of the breast: a rare case report Int J Surg Case Rep 14 141-145 https://doi.org/10.1016/j. ijscr.2015.07.036 PMCID: 4 573862

12. Karvouni E, Papakonstantinou K, and Dimopoulou C, et al (2009) Abnormal uterine bleeding as a presentation of metastatic breast disease in a patient with advanced breast cancer Arch Gynecol Obstet 279(2) 199-201 https://doi.org/10.1007/s00404-008-0665-9

13. Meydanli MM, Karadag N, and Ataoglu O, et al (2002) Uterine metastasis from infiltrating ductal carcinoma of breast in a patient receiving tamoxifen Breast 11(4) 353-356 https://doi.org/10.1054/brst.2002.0447 